Afroditi Zartaloudi $^{1}$

\title{
529 - Suicide in elderly people
}

${ }^{1}$ University of West Attica, Athens, Greece

Background: Suicide is a tragic and distressing phenomenon. The problem of suicide in late life is often neglected by medical professionals, policy makers and the general public. Suicidal behavior in older adults (65 years old and over) is a major public health issue in many countries. Suicide rates are highest among the elderly.

Objective: To investigate the main factors associated with suicidal ideation, attempts and completed suicide among the elderly.

Method: A literature review was carried out in PubMed and Scopus database.

Results: Depression is the most relevant cause found, combined with chronic physical suffering, loss, bereavement, abandonment, loneliness, family conflicts and social exclusion. Differences in gender, ethnicity, the ageing process, social issues and cultural backgrounds are also major contributing factors. The major causal factors for attempted suicide are degenerative and chronic diseases, physical dependence and disability, physical and psychological pain, mental and neurocognitive disturbances and suffering. The issue of suicide prevention in the elderly is also addressed.

Conclusions: The negative effects on families, friends and communities following a suicide reinforce the urgency for a better understanding and prevention of suicide. Suicide associated with depression in the elderly can be prevented, provided the person is properly treated. Innovative strategies should improve resilience and positive aging, engage family and community support networks, reach vulnerable older adults, and promote health professionals' knowledge on elderly suicide.

\section{0 - The effects of lithium and inflammation on the atherosclerosis of older bipolar patients at high risk for cardiovascular disease \\ Shang-Ying Tsai, Pao-Huan Chen, Kuo-Hsuan Chung}

Department of Psychiatry, Taipei Medical University and Taipei Medical University Hospital, Taipei, Taiwan

Background: Atherosclerosis can result in serious cardiovascular disease (CVD) and is associated with inflammation and psychopharmacological treatment in bipolar disorder. We attempt to investigate the effects of lithium and inflammation on the atherosclerotic development in older bipolar adults at high risk for cardiovascular disease.

Methods: The euthymic out-patients with bipolar I disorder aged over 45 years and concurrent endocrine or cardiovascular disease were recruited to measure their bilateral carotid intima media thickness (CIMT) and circulating levels of lithium, valproate, sTNF-R1, sIL-6R, and lipid profile. All clinical information were obtained by directly interviewing patients and reviewing all medical records.

Results: Forty eight patients with mean 48.3 years old and mean 27.2 years of age at illness onset were recruited. After controlling for the body mass index, multivariate regression analyses showed that older age, lower lithium level, and higher plasma sTNF-R1 level were associated with higher CIMT and collectively accounting for $33.1 \%$ of the variance in CIMT. Blood level of low density lipid or valproate has none relationship with CIMT. 